Monatsschrift f. Geburtshülfe u. Gynäkologie 1935;99:I-IV

\title{
Contents, Vol. 99, 1935
}

Inhaltsverzeichnis.

Origínalarbeîte $\pi$ Seite

Bak, Michael, Die Bewertung der Fingerabdrüeke von Zwillingen . . 271 Chmelewsky, W. N., Behandlung der spitzen Kondylome mit der

Quarzlampe 177

Dienst, Arthur, Über die Ätiologie und Pathogenese der Eklainpsie mit

besonderer Berücksichtigung der Beschaffenheit des Herzens . . 13 Ehrhardt, Karl, Ein weiterer

Beitrag zuni Vorkommen des Corpus-

luteum-Hormons 257

Eufinger, II., und W. Kiltz, Der Einfluß von Narkose und Operation

auf die Zahl und Funktion der Leukozyten 279

- und II. Krupp, Dermographismus in der Gestationsperiode . . 343 Fekete, Alexander v., Über präinenstruelle Temperaturerhöhungen. . 29 Fischer, Erich, Kritisehe Untersuehungen über Sehwangerschafts-

reaktionen 69

Fraijmann, S. A., Über die Kornplikation der Gravidität und Geburt

dureh Myelitis 210

Goecke, II'., Die Bedeutung der Höcnst > vehenzaMen beiin vorzeitigen

Blasensprung unter besonderer Berücksichtigung der Geburt bei

alten Erstsebärenden mit normal weitein Beeken 24

Goldmaier, Erich, Über die Vererbung von Mißbildungen

Hartung, Werner, siehe Spiegler, Rudotf.

Hülsmeyer, Paul, Über einen Fall von juveniler Blutung bei Thrombopenie 225

Jaschke, Rud, Th. v., Ein einfaches $\Gamma$ VIittel zur Sicherung des Sterilisierungscîfektes 1

Jegoroff, B. A., siehe Sserdjukoff, M. G.

Junghans, Erich, Über Tbrombosen und Embolien an der Universitäts-

Frauenklinik in Halle a. d. S 134

Karlin, Max, und Alexandra Osjakina, Zur Pathologie und Klinik

der ortsfremden endometrioiden Wueherungen 353

Kelemen, Stephan, Beiträge zu den paralytischen Gehimstörungen

vvährend der Gestation und zur Frage der mit Herzfehler koinpli-

zierten Selnvangersehaft $\quad 140$

Kiltz, W., siehe Eufinger, II.

Klein, S. M., Zur Therapie der Placenta praevia $\quad 170$

Krupp, II., siehe Eufinger, II.

Kuschelewsky, A. P., Der Wert der Konfrontation und der Bordet-

Genii'ouselien Methode 1'ür die Erkennung der latenten Gonorrhoe- 
Erkrankung der Frau 73

Logwinsky, P. X., Über postkliniakterische Blutungen 286

M-enzel, Ulrike, Metastasen bei P«erperalsepsis 204

Mosettig, Egon, Ein Fall von Thorakopagus bei Tubargravidität ... 164

Müller, Ilans-Gert, Einseitiger Krukenbergtumor 348

Osjakina, Alexandra, siehe Karlin, Max

Presses, Heinz, Zur Beurteilung der Höchstweheüzahl 260

Puhr, Ludwig, Beitrag zur Pathogenese des Krukenbergtumors . . . 229 Rhemann, Franz,

Beiträue zur Frage der Myomenukleation während

der Gestation 298

Schumacher, Paul II., Zur Frage der periodischen Unfruehtbarkeit

des Weibes 64

Seiíz, L., Zur Ausschälung der Myome

Alle Rechte vorbehalten

Pierersoh $\theta$ Hofbuchdruckerei Stephan Geibel \& Co. in Altenburg, Thür.

yy Inhaltsverzeichnis.

Seit $\beta$

Seitz, L., Über hypophyse Störuiigen im Ansehluß an Scl $\pi$ vanger-

schaft und Geburt 321

Spiegler, Rudolf, und WernerHarlung, Gonorrhoe und Sch $>$ vangersehaft 41

Sserdjukoff, M. G., und B. A. Jegoroff, Über die Entstehung von

vielfachen Venenthrombosen als allergische Reaktion des venösen

Systems beim Krebs der Genitalien 56

Sláhler, Frilz, Über 1ST Гälle von puerperaler Sepsis und Pyämie, na $\Varangle \cdot h$

einein 12jährigen Beobaehtungsinaterial der Ui $\pi$ versitäts-Frauen-

klinik Frankfurt a. 31193

Winkler, Hellmuth, Die Orasthinanspreehbarkeit des Dickdarms. Ein

Test für don funktionellen Zustand des Uterus 152

Winter, Egon Werner, Der vorzeu $1 / 8 \mathrm{se}$ und sehr îrühzeitige Blasen-

sprung, seine Pathogenese und Therapie 332

Wolkow, J. N·, Weitere Beobaehtungen über die Therapie der Eklanipsie 290

Gre zzgebiete der Geburtshilfe und Gynäkologie.

Narkose, Anästhesie und Däminersehlaf. Übersicht über das Schrifttum

des Jahres 1934. Yon Dr. Herbert Buschbeck-Würzburg . 82

Cholezystitis. Cholelithiasis und Appendizitis. Yon Dr. Eugen Kulka,

Prag 89

Der Stoffweehsel bei Sel $\pi$ vaimeren. Y. Jahresübersieht. Von Dr. phïl.

Dr. med. O. Mühlbock, Berlin

183

Physikalische Heilmethoden einsehließlich der Hydrotherapie. Yon

Hermann Tranken, Freiburg i. Br 240

1st die Schwangerschaftsunterbrechung' auf Grund eugenischer Indi-

kation bereits nach geltendem ßecht zulässig.' Yon Landgerichts-

rat i. R. Dr. jur. Franz Nerrkamp, Bielefeld 243

Die Erkrankuneen der Leber, der $\Lambda$ iere und der ableitenden Harmvege

in ihren Beziehangen zur Geburtshilfe und Gynäkologie. Yon

Privatdozent Dr. Hans Rupp, Bonn a. Rh 305 
Kreislaufberieht für das Jahr 193\%3/8. Yon H. Reichenmiller, Tubingen 360 Sitzungsberichte aus geburtshilflich-gynäkologischen Gesellschaften. Gynäkologisehe Gesellsehaft zu Breslau. Sitzung vom 20. Nov. 193493 Gynäkoloiíische Gesellsehaft zu Breslau. Sitzung vom 15. Jan. 1935374 Gesellsehaft für Geburtshilfe und Gynäkologie Berlin. Sitzung vom 19. Okt, 1934. Sitzung vom 23. Nov. 1934107

Gesellsehaft für Geburtshilfe und Gynäkologie zu Berlin. Sitzungs berichte vom 18. Januar und 15. Februar 1935 251

59. Tagung der Deutsehen Gesellsehaft für Chirurgie. Berlin 24. bis

27. April 1935. Referent: Dr. L. Waldeyer, Berlin 368

Mittelrheinische Gesellsehaft für Geburtshilfe und Gyiiäkoloi > ie.

93. Sitzung am 16. Dezember 1934 zu Frankfurt a. II. ... 97

Geburtshilflieh-gynäkolo $7 / 8$ isehe Gesellsehaft in Wien. Sitzungen vom

27. November, 11. Dezember 1934, 15. Januar 1935121

--, Sitzung vom 12. Februar 1935318

Buchbespreeh $\pi$ nííen $124,256.378$

Personalien und Tagesnachriehten 128, 256, 380

In memoriam Hofrat Prof. Dr. Emil Knauer 379 